\title{
Interactions avec le milieu et développements récents dans l'emploi des traceurs artificiels
}

\author{
PAR
}

\section{J. Molinari}

Ingénieur au Commissariat à l'Energie Atomique

Service d'Applications des Radioéléments et des Rayonnements C.E.N. Grenolble

\section{Evolution de l'emploi des traceurs}

Si l'usage des traceurs en hydrologie remonte à un siècle (*), il s'est pratiquement restreint aú karstjusqu'à une époque très récente.

Ces expériences, limitées à une mise en évidence de circulations souterraines étaient effectuées à l'origine au moyen de diverses substances, puis, presque exclusivement ensuite, à l'aide d'uranine (fluoresceine sodique) d'une mise en oeuvre incomparablement plus aisée (Paloc, 1975). Leur aspect spectaculaire comme leur indéniable simplicité popularisèrent très tôt ce type d'opérations de marquage et la notion de traceur.

Ce n'est pourtant qu'après la fin de la seconde guerre mondiale que s'étendit le domaine d'application des traceurs. Un foisonnement d'études, motivées par le désir de ménager aux énormes investissements nucléaires des applications diversifiées, suscita alors chez bon nombre de physiciens des vocations d'hydrologue. Si leurs expériences de substitution de traceurs radioactifs aux traceurs utilisés jusque là ne furent pas toujours convaincantes, on doit à ces chercheurs la révélation des isotopes et des radioisotopes du milieu dont l'interprétation familière aujourd'hui aux hydrogéologues, leur a ouvert des voies nouvelles et extrêmement fructueuses.

En fait, c'est aux praticiens de l'hydrométrie que l'on doit le développement rationnel des applications de traceurs. Les méthodes de mesure des débits de liquides imaginées en France dès $1863\left(^{* *}\right)$, méthodes qui impliquent un certain nombre d'exigences quant à la qualité des traceurs, à la préci-

(*) Selon Paloc (1975) la première expérience rigoureuse aurait été réalisée en 1877 par Knopp et Ten Brink à l'aide de 10 tonnes de chlorure de sodium et de 10 kilogs de fluoresceine.

(**) Cité par André (1964) sion des mesures et à la rigueur de l'expérimentation, n'ont pris un réel essor qu'après 1950, avec leur systématisation, par Electricité de France (André 1969).

Ce sont précisément les problèmes méthodologiques liés à l'interprétation de certains aspects de ces mesures qui favorisèrent, grâce à quelques rares laboratoires situés à mi chemin de la pratique de l'hydrologie et de celles de l'industrie chimique, une osmose bénéfique des mathématiques du traitement $\mathrm{du}$ signal et de la méthodologie des transports de masse vers les techniques hydrologiques.

Ces récents progrès, présentés dans l'exposé de MM. Guízerix et Margrita, en conférant une rigueur plus grande aux applications de traceurs, incitaient à un réexamen critique de leurs conditions d'emploi.

\section{Les exigences nouvelles en matière de traçage}

L'information la plus complète que l'on puisse souhaiter obtenir pour l'étude de propagation de fluides, dans un système quel qu'il soit, est la réponse impulsionnelle de ce système à un stimulus unitaire d'entrée.

Il ressort de l'exposé méthodologique qui précède, que l'exploitation des signaux fournis par des traceurs naturels ou artificiels conduit à l'obtention de distributions des temps de séjour qui sont précisément des réponses impulsionnelles particulières.

L'analyse de ces distributions des temps de séjour (D.T.S.) à la lumière de modèles mathématiques de propagation permet de cerner l'allure de processus se manifestant à l'intérieur de systèmes, lesquels en hydrologie souterraine notamment, sont souvent inaccessibles.

La qualité aujourd'hui attendue de ce type d'informations, comme la précision des bilans de traceurs exigée pour les me- 
sures de débits, impose de disposer de traceurs les plus représentatifs possibles du milieu étudié.

De telles préoccupations n'avaient guère embarrassé les premiers praticiens du marquage qui, peu soucieux de la rigueur de leurs observations, n'hésitèrent pas à recourir, pour marquer l'eau, à des substances allant de la sciure de bois à divers corps flottables en passant par des substances plus banales telles l'avoine ou le son (in Paloc 1975).

Bien que, comme nous le verrons plus loin, d'aussi surprenants indicateurs n'aient pas qu'un intérêt anecdotique et continuent a être en usage pour des besoins très spécifiques, il n'en est pas moins vrai que le souci essentiel qui prévaut en hydrologie est la représentativité des traceurs de l'eau, le "bon traceur" devant être facilement véhiculé par l'eau et ne suivre qu'elle, sans altération ni modification de ses propriétés spécifiques.

Cette recherche de la perfection, qui occupait récemment encore bon nombre d'expérimentateurs, ne peut aboutir puisque, c'est en quelque sorte le paradoxe du marquage, toute identification résulte nécessairement d'une propriété spécifique distinguant le traceur du milieu qu'il est censé représenter.

En l'absence de solution idéale, on tiendra le plus grand compte, pour orienter son choix, du milieu dans lequel évoluera le traceur et des types d'interactions auxquels il risque d'être soumis.

\section{Influence de la nature du milieu sur le comportement de substances traçantes}

Même en l'absence d'intervention humaine l'eau dans la nature est toujours un milieu complexe où coexistent :

- des êtres vivants du règne végétal ou animal (phyto et zooplanctons)

- des sédiments en suspension

- des colloides

- et d'innombrables ions.

En outre, en hydrologie souterraine et, à des degrés moindres, en hydrologie karstique et en hydrologie de surface, l'eau dont on étudie le mouvement se trouve en contact plus ou moins intime avec des matériaux extrêmement divers.

Ainsi le comportement du traceur sera non seulement affecté par la composition du milieu aqueux mais aussi par le parcours de ces eaux:

- en hydrologie de surface et en hydrologie karstique où interviennent la forme et la nature des berges, du lit et des chenaux;

- en hydrologie souterraine à perméabilité en petit (ou d'interstice) où les surfaces de contact et les temps de séjour peuvent atteindre des valeurs extrêmement élevées ;

- en zone non saturée, en particulier dans les sols, domaines d'intenses activités chimiques et biologiques où l'écoulement n'est jamais permanent et les risques de fixation accrus.

\section{Les principaux mécanismes d'interaction}

Alors que pour les eaux de surface, océans, lacs, grands réservoirs, cours d'eau et circulations karstiques, le choix des traceurs présente généralement peu de difficultés (à l'excep- tion toutefois des milieux fortement pollués), ce choix est extrêmement délicat pour les eaux souterraines circulant dans des terrains poreux, particulièrement en porosités d'interstices, où les surfaces de contact eau-terrain sont importantes.

Dans tous les cas ce choix découle des contraintes imposées par les différents processus d'interaction susceptibles d'intervenir (Molinari 1969b). Ces interactions peuvent être rangées en 3 classes:

"substances de l'eau" - "substratum",

"substances" -.. "substances",

"substances" "biomasse".

4.1. Interactions des substances contenues dans l'eau avec le substratum

- La filtration, processus purement mécanique qui n'affecte que colloïdes, matières en suspension et sédiments.

- L'adsorption physique, dans laquelle la liaison entre le corps adsorbé et l'adsorbant est faible, du type Van der Waals.

- L'adsorption chimique (ou chimisorption), qui met en jeu des liaisons plus fortes, du type covalent.

- L'échange d'ions, qui est un phénomène d'équilibre entre des ions déjà fixés (électrovalence) par l'échangeur et ceux de la solution de contact.

- La précipitation colloïale, notamment en présence des complexes argilo-humiques du sol, dont une fraction se présente à l'état très fin. Le caractère hygroscopique de ces composés favorise la formation de gels par gonflement.

Rappelons que les propriétés de ces complexes donnent la possibilité d'adsorber de grandes quantités de substances en solution, ce qui leur confère un pouvoir de rétention redoutable.

\subsection{Interactions mutuelles des substances en solution}

- La précipitation chimique, liée à la solubilité des substances en phase aqueuse; une modification faible du milieu peut la provoquer (apparition d'un liquide précipitant, addition d'un électrolyte, changement de $\mathrm{pH}$.)

- Les altérations chimiques, essentiellement d'oxydo-réduction ou de complexation : la plupart des réactions d'oxydation sont des auto-oxydations dans lesquelles le seul agent oxydant est l'oxygène moléculaire. L'eau renferme des quantités suffisantes d'oxygène pour amorcer les processus d'auto-oxydation, mais ensuite la décomposition se fait en chaine et reste pratiquement indépendante de la concentration en oxygène.

Parfois il suffit d'une trace d'un métal possédant un ou plusieurs degrés d'oxydation pour catalyser une réaction d'oxydoréduction. La lumière peut aussi fournir l'activation nécessaire à de telles réactions.

\subsection{Interactions des substances en phase aqueuse avec la biomasse}

- La métabolisation due à l'activité biologique des microorganismes (algues, zooplancton, phytoplancton). Dans les sols, elle sera surtout efficace dans les premiers décimètres de la surface, riches en humus et permettant la survie de bactéries aérobies.

\section{Exemples de sorption}

Tous ces mécanismes de rétention se manifestent, à des degrěs divers, lorsque les traceurs sont en contact avec le terrain ou des matières organiques. Nous choisirons pour exemples les plus caractéristiques celui des argiles et celui des humus. 


\subsection{La rétention des traceurs sur les argiles}

Toutes les argiles se présentent sous l'aspect d'innombrables grains fins, de forme aplatie et de diamètre inférieur à 2 microns. Ces argiles sont des aluminosilicates hydratés dont la structure est fort complexe. Elles sont constituées d'empilements de couches bidimensionnelles d'aluminosilicates : les feuillets. Les faces de ces feuillets sont chargées négativement. Tandis que la cohésion du feuillet élémentaire est de nature ionique, ce qui lui confère une grande stabilité en raison de la force de ces liaisons, la cohésion de l'édifice est assurée par des liaisons de faible énergie fournies par l'eau et les cations insérés entre les feuillets.

La rétention de traceurs sur un tel matériau peut être attribuée à la fois à tous les mécanismes déjà invoqués

- l'adsorption physique, à la faveur de la structure et de la dimension des grains ;

- la chimisorption, facilitée par la charge ionique des feuillets ;

- la précipitation, favorisée par l'état colloïdal du matériau.

Mais le mécanisme de rétention prépondérant résulte essentiellement de la présence de cations échangeables, soit en bordure des feuillets, soit dans l'espace qui sépare ces feuillets, (cf. figure 1), ce qui confère à ce matériau une capacité d'échange cationique extrêmement élevée comme l'indiquent quelques valeurs citées dans le tableau 1 (in Molinari 1969 b)
5.2. La rétention des traceurs sur les collö̈des du sol

Seuls, les colloïdes électronégatifs ou acidoïdes sont abondants dans le sol. Ce sont, d'une part les argiles, d'autre part des composés humiques.

Rappelons que le sol renferme généralement dans ses couches superficielies 2 à $5 \%$ de matière organique (ce pourcentage peut s'élever quelquefois à des valeurs de l'ordre de $20 \%$ ). On désigne communément ces éléments organiques par le terme général d'humus. En fait, ces éléments organiques comprennent une fraction à structure fibreuse encore organiscée et une fraction à l'état très fin, colloïdal. C'est cette dernière fraction qui constitue l'humus proprement dit.

Les composés humiques sont des colloïdes hydrophiles ou émulsoïdes qui s'entourent d'une épaisse couche d'eau de solvatation (cf. figure 2 ).

Ce caractère hygroscopique, joint à leurs propriétés d'acide faible, donne aux composés humiques la possibilité d'adsorber de grandes quantités de cations échangeables ce qui leur confère un pouvoir d'échange redoutable. Ainsi on attribue aux acides humiques des capacités d'échange cationique de 300 à 400 milliéquivalents par 100 grammes.

Les complexes argilo-humiques, qui cumulent les capacités d'échange de deux constituants ont des capacités d'échange intermédiaires entre celles des humus et celles des argiles (200 à 300 milliéquivalents par 100 grammes).

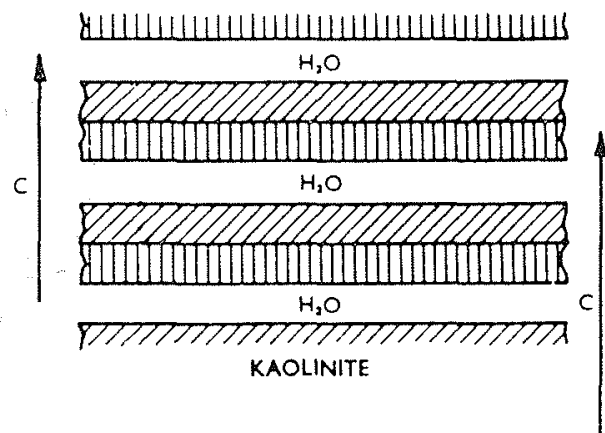

1/ Représentation schématique des structures en couches caractéristiques de certains types d'argiles. La flèche indique l'axe du cristal (d'après C.B. Amphlett in Molinari 1969 b)

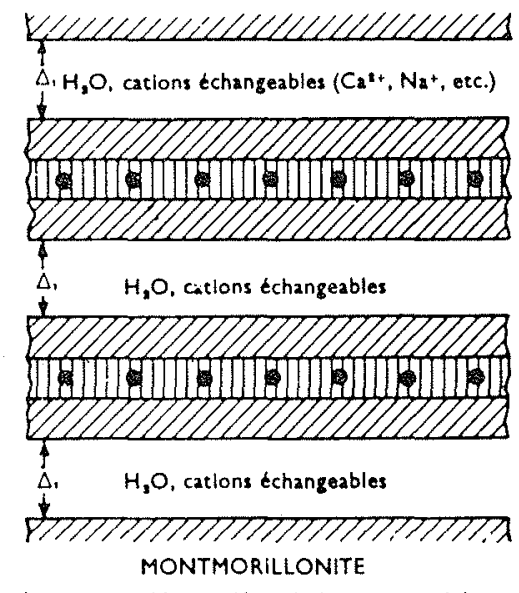

$\left(\triangle_{1}\right.$ est variable et dépend des cations echangeables presents)

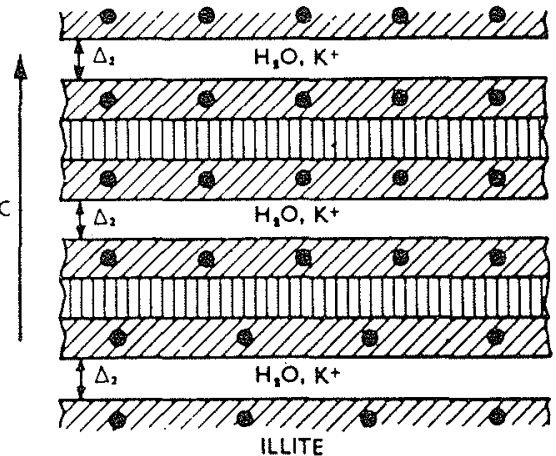

$\left(\Lambda_{2}\right.$ ne varie que peu, est en genéral plus petit que $\left.\Delta_{1}\right)$

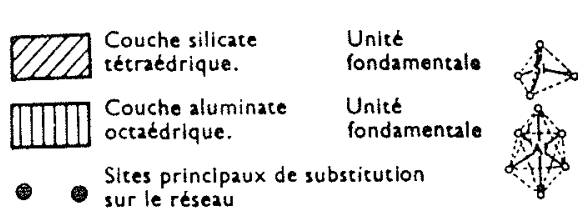

Tableau 1

Capacité d'échange de quelques argiles

(d'après C.B. Amphlett in Molinari 1969 b

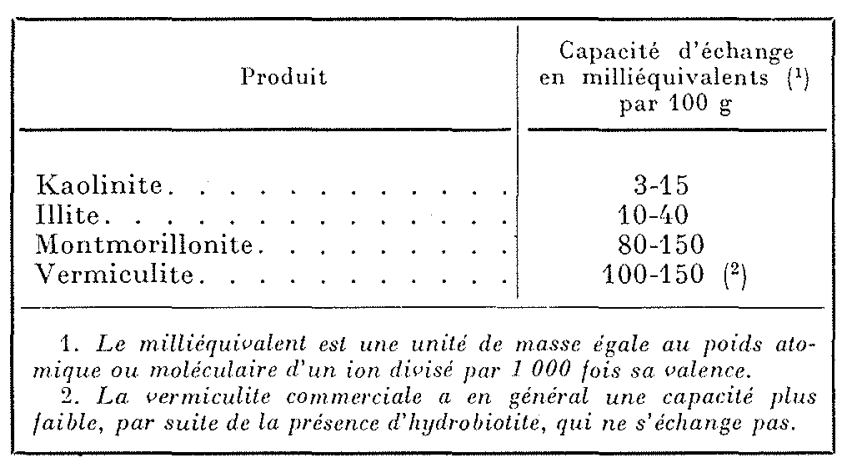

Colloüde électronégatit

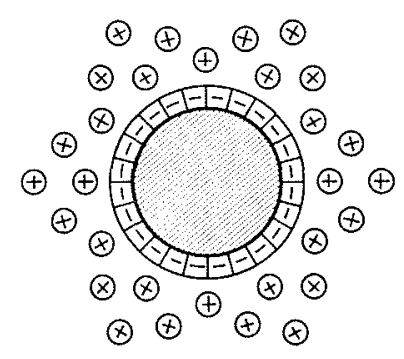

2/ Schéma de particules colloïdales
Collö̈de électropositif

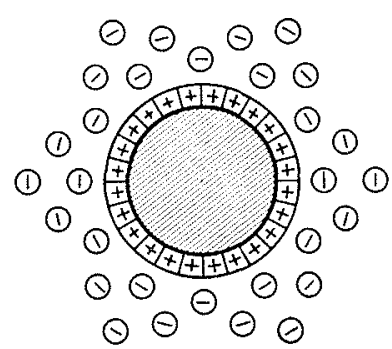




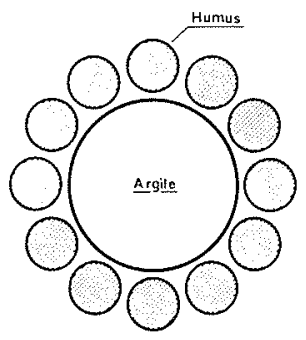

3/ Schéma du complexe argilo-humique

\section{Propriétés physicochimiques requises pour un traceur de l'eau}

Hormis le cas où des substances macroscopiques s'imposent pour simuler le devenir de matériaux grossiers ou pour, comme nous le montrerons dans les applications au Génie Civil, localiser des interfaces entre milieu ouvert et milieu poreux, on évite aujourd'hui l'emploi de traceurs non miscibles, en raison de l'importance des risques de ségrégation par filtration, sédimentation ou flottation.

On évitera pour des raisons analogues les molécules à important encombrement stérique que leur faible solubilité repousse de la phase aqueuse vers le substrat où elles se fixent généralement par physisorption.

Ces macromolécules exclues, les molécules moins complexes et les ions sont caractérisés par la présence de charges électriques jouant un rôle important dans les processus d'interaction où l'échange d'ions est le plus à craindre, en hydrologie souterraine surtout, les sols contenant pour la plupart d'entre eux, des minéraux argileux mêlés à des produits organiques.

L'échange cationique étant, comme on l'a montré, largement prépondérant dans la plupart des milieux, il en résulte que les meilleurs traceurs miscibles seront des anions ou des complexes faiblement ioniques.

A ces processus de sorption prépondérants peuvent s'ajouter des agressions chimiques ou même biologiques dont l'action quelquefois conjuguée peut exclure des traceurs satisfaisant aux règles précédentes. C'est le cas en particulier des traceurs organiques ou organométalliques dont la fragilité $\left(^{*}\right)$ peut en proscrire l'emploi dans certaines conditions. Ces dernières raisons conduisent à préférer dans les cas les plus difficiles, notamment en hydrologie souterraine, l'emploi de traceurs salins anioniques.

On dispose enfin, en dernier recours, d'une catégorie exceptionnelle de traceurs constituée par les variétés isotopiques de la molécule d'eau. De tels traceurs, qui présentent en principe les mêmes propriétés chimiques que la phase qu'ils caractérisent, sont qualifiés de traceurs intrinsèques. (Molinari 1969 b).

$\mathrm{Si}$, comme M. Fontes le montrera dans l'exposé qui suit, on tire un grand parti des variations naturelles des trois variétés isotopiques principales de l'eau, DHO, HTO et $\mathrm{H}_{2}{ }^{18} \mathrm{O}$, le seul traceur artificiel intrinsèque de l'eau d'emploi courant est l'eau tritiée HTO dont l'indiscutable effet de retard en milieu poreux (par échange isotopique avec l'eau du milieu) confirme l'irréalisme de la notion de marqueur idéal.

(*) Comme en témoigne la sensibilité de l'uranine à la photo-dégradation et l'instabilité de certains complexes en présence d'autres ions.

\section{Autres critères de sélection de traceurs artificiels}

L'énoncé de ces règles générales doit être bien entendu assorti de nombreuses exceptions qui n'étonneront point les praticiens familiarisés avec la désarçonnante complexité des interactions évoquées. Ainsi, les argiles peuvent être le siège d'échanges anioniques (Rochon 1976) et, dans certaines circonstances, des traceurs cationiques se sont révélés préférables aux traceurs anioniques.

Ces exceptions ne sont pas seules à justifier de la diversité des traceurs aujourd'hui en usage, car, même dans des milieux où d'importantes interactions sont à craindre, des contraintes technologiques, analytiques ou financières relèguent au second plan la qualité du marquage.

Ces contraintes sont de tous ordres :

- d'ordre technologique, en premier lieu, le traceur ne pouvant être naturellement présent dans le milieu qu'à des concentrations suffisamment faibles pour ne pas imposer la mise en oeuvre de quantités rédhibitoires. Pour les mêmes raisons, sa sensibilité de détection doit être la plus grande possible.

- d'ordre socioéconomique, la toxicité du traceur doit être nulle ou tout au moins négligeable et son coût, comme celui de son analyse, doit être le plus faible possible.

\section{Les récents acquis analytiques}

Il est indubitable que les premiers travaux sur les traceurs furent essentiellement axés sur les techniques d'identification. La très ancienne popularité de l'uranine doit être davantage attribuée à sa facilité de détection (à une concentration de $10^{-7} \mathrm{~g} / \mathrm{g}$ par simple observation) qu'à son excellent comportement dû à son caractère anionique marqué en milieu neutre.

Cette approche "analytique" qui a pris tout son essort dans l'immédiat après guerre a entretenu l'existence d'un certain nombre d" "écoles" s'appuyant généralement toutes sur un laboratoire spécialisé dans une technique d'identification particulière.

Si certaines études sur l'utilisation de traceurs biologiques (bactéries serratia marcescens Dombrowski 1970), de traceurs organoleptiques (dipentène, acétate d'isobornyle, salicylate d'isoamyle - Schnitzer et Wagner 1969), de traceurs photochromiques décelés par excitation laser (dinitrobenzine pyridine - Frantisak et all 1969) n'ont pas encore fait l'objet d'applications de routine, des investigations persévérantes ont abouti pour l'école autrichienne, spécialisée dans l'étude du karst, à l'abandon des traceurs solides autres que les spores de lycopodes diversement colorés avant d'être mis en œuvre puis recueillis et dénombrés sur des filets à plancton.

L'efficacité de ces derniers traceurs, dont les utilisateurs parviennent à obtenir des courbes de restitution (Batsche et all . 1966), est évidemment limitée aux karst où pollens et microfaune sont d'ailleurs exploités avec succès comme indicateurs naturels (Rouch 1972).

Divers laboratoires spécialisés dans la mesure de traceurs fluorescents ont pris la relève des spéléologues qui ne tiraient pas le meilleur parti de l'uranine alors mesurée par colorimétrie. La substitution de traceurs fluorescents (uranine, rhoda- 
mines, sulforhodamines) aux quelques traceurs colorés utilisés sporadiquement jusqu'alors (bleu de méthylène, fuschine acide, vert acide, phénolphtaléine) ont amélioré d'un facteur mille à dix mille la sensibilité de détection des traceurs colorés (concentration minimale détectable de l'ordre de $10^{-11} \mathrm{~g} / \mathrm{g}$ ) leur ouvrant ainsi, pour ceux d'entre eux dont la forme ionique est favorable, d'autres domaines que celui du karst et de l'hydrologie de surface, et ce, a une échelle encore inaccessible aux autres traceurs (Molinari 1969 a)

Mais c'est sans aucun doute avec les techniques nucléaires que les investigations ont été le plus systématiquement conduites. Ces travaux sérieusement épaulés par d'importantes études sur les risques de propagation de rejets radioactifs, ont vulgarisé outre l'utilisation d'eau tritiée, d'intéressants traceurs salins anioniques à vie courte $\left({ }^{82} \mathrm{Br},{ }^{1}{ }^{1} \mathrm{I}\right)$, suppléé à l'absence de traceurs anioniques à vie longue par la mise au point de cations radioactifs chélatés $\left({ }^{5} \mathrm{Cr}\right.$ EDTA, et, entre autres, différents complexes de ${ }^{60} \mathrm{CO},{ }^{59} \mathrm{CO}$ et de ${ }^{124} \mathrm{Sb}$ ), et développé l'emploi de générateurs isotopiques (notamment ${ }^{11^{3}} \mathrm{Sn}$, ${ }^{113} \mathrm{In}$ et ${ }^{137} \mathrm{Cs},{ }^{137} \mathrm{Br}$ ). susceptibles de libérer, à la demande, des radiotraceurs éventuellement chélatés sous une forme anionique.

L'utilisation de ces traceurs qui ne bénéficiaient pas d'une sensibilité de détection considérable (contrairement à une opinion généralement répandue), a surtout été entravée par les difficultés de mise en oeuvre, confinant désormais les radiotraceurs à des applications extrèmement spécifiques dont des exemples seront donnés au cours d'autres exposés.

L'analyse chimique traditionnelle et ses prolongements perfectionnés semblent avoir contribué plus modestement aux

Tableau 2

Caractéristiques et domaines d'utilisation des traceurs artificiels de l'eau les plus couramment employés

\begin{tabular}{|c|c|c|c|c|c|c|c|c|}
\hline Désignation & Forme chimique & Forme ionique & \multicolumn{2}{|c|}{ Procédé de mesure } & $\begin{array}{l}\text { Technologie de } \\
\text { mesure et con- } \\
\text { ditions opéra- } \\
\text { toires (volume } \\
\text { d'échantillon) }\end{array}$ & $\begin{array}{l}\text { Concentration } \\
\text { minimale dé- } \\
\text { tectable }\end{array}$ & $\begin{array}{l}\text { Coüt d'une } \\
\text { mesure } \\
\text { (estimations } \\
1973 \text { ) }\end{array}$ & Domaine d'utilisation \\
\hline $3_{\mathrm{H} \text { (tritium) }}[1]$ & $\begin{array}{l}\text { Eau tritiée } \\
\text { (HTO) }\end{array}$ & $\begin{array}{l}\text { Traceur intrin- } \\
\text { sèque de l'eau }\end{array}$ & \multicolumn{2}{|c|}{$\begin{array}{l}\text { Radioisotope période } 12,3 \text { ans } \\
\text { Emetteur béta mesuré par comp- } \\
\text { tage gazeux ou scintillation li- } \\
\text { quide }\end{array}$} & $\begin{array}{l}\text { Laboratoire } \\
\text { Mesure directe } \\
\text { ou par recon- } \\
\text { centration élec- } \\
\text { trolytique }(1 \text { à } \\
1000 \mathrm{ml})\end{array}$ & $\begin{array}{l}\text { Quelques unités } \\
\text { Tritium (UT) } \\
\text { (1 UT }=3,2 \\
\text { picoCi/litre) }\end{array}$ & 60 à 300 francs & $\begin{array}{l}\text { Traceur universel dont } \\
\text { l'emploi est restreint } \\
\text { par les risques d'inter- } \\
\text { férences avec les me- } \\
\text { sure d'environnement } \\
\text { de tritium naturel }\end{array}$ \\
\hline Chrome 51 [1] & $\begin{array}{l}\text { Versénate } \\
\text { (EDTA) }\end{array}$ & $\begin{array}{l}\text { Cation chélaté } \\
\text { sous forme } \\
\text { anionique }\end{array}$ & \multirow{3}{*}{ 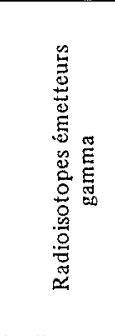 } & Période 28 jours & \multirow{3}{*}{ 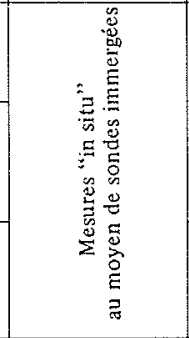 } & $30 \mu \mathrm{Ci} / \mathrm{m}^{3}$ & \multirow{3}{*}{ 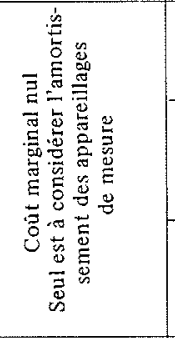 } & $\begin{array}{l}\text { Hydrologie souter- } \\
\text { raine }\end{array}$ \\
\hline Brome 82 [1] & $\begin{array}{l}\text { Bromure } \\
\text { d'ammonium }\end{array}$ & Anion & & Période $36 \mathrm{~h}$. & & $1 \mu \mathrm{Ci} / \mathrm{m}^{3}$ & & $\begin{array}{l}\text { Tous domaines auto- } \\
\text { risés parl'extrême briè- } \\
\text { veté de la période ra- } \\
\text { dioactive }\end{array}$ \\
\hline Iode $131[1]$ & $\begin{array}{l}\text { Iodure de so- } \\
\text { dium }\end{array}$ & Anion & & Période 8 jours & & $4 \mu \mathrm{Ci} / \mathrm{m}^{3}$ & & $\begin{array}{l}\text { Tous domaines auto- } \\
\text { risés par la relative } \\
\text { brièveté de la période } \\
\text { radioactive }\end{array}$ \\
\hline \multirow{2}{*}{ Iodure [2] } & \multirow{2}{*}{$\begin{array}{l}\text { Iodure de so- } \\
\text { dium }\end{array}$} & \multirow{2}{*}{ Anion } & \multicolumn{2}{|c|}{$\begin{array}{l}\text { a) Microdosage cataly tique au } \\
\text { moyen d'un analyseur }\end{array}$} & $\begin{array}{l}\text { Laboratoire } \\
\text { (quelques milli- } \\
\text { litres) }\end{array}$ & \multirow{2}{*}{$10^{-9}$} & 10 francs & \multirow{2}{*}{ Traceur universel } \\
\hline & & & \multicolumn{2}{|c|}{$\begin{array}{l}\text { b) Analyse par activation (dosage } \\
\text { spécifique) }\end{array}$} & $\begin{array}{l}\text { Laboratoire nu- } \\
\text { cléaire (quel- } \\
\text { ques millilitres) }\end{array}$ & & 60 francs & \\
\hline \multirow{2}{*}{ Bichromate [2] } & \multirow{2}{*}{$\begin{array}{l}\text { Dichromate } \\
\text { de sodium }\end{array}$} & \multirow{2}{*}{ Anion fragile } & \multirow{2}{*}{\multicolumn{2}{|c|}{$\begin{array}{l}\text { Colorimétrie à la diphénylcarba- } \\
\text { zide }\end{array}$}} & \multirow{2}{*}{$\begin{array}{l}\text { Laboratoire } \\
\text { sommaire (quel- } \\
\text { ques millilitres) }\end{array}$} & $10^{-6}$ & 10 francs & \multirow{2}{*}{ Hydrologie de surface } \\
\hline & & & & & & $\begin{array}{l}10^{-8} \text { avec re- } \\
\text { concentration }\end{array}$ & 50 francs & \\
\hline Indium [1] & $\begin{array}{l}\text { Versenate } \\
\text { (DTPA) }\end{array}$ & $\begin{array}{l}\text { Cation chélaté } \\
\text { sous forme } \\
\text { ionique }\end{array}$ & \multicolumn{2}{|c|}{$\begin{array}{l}\text { Analyse par activation neutro- } \\
\text { nique }\end{array}$} & $\begin{array}{l}\text { Laboratoire } \\
\text { nucléaire } \\
\text { (quelques milli- } \\
\text { litres) }\end{array}$ & $10^{-10}$ & 100 francs & $\begin{array}{l}\text { Hydrologie souter- } \\
\text { raine - Utilisation } \\
\text { restreinte à des cas } \\
\text { particuliers en raison } \\
\text { des coūts élevés du } \\
\text { traceur et des analyses }\end{array}$ \\
\hline $\begin{array}{l}\text { Uranine } \\
\text { (fluoresceine } \\
\text { sodique ou } \\
\text { hydraulique) }\end{array}$ & \multirow{5}{*}{ 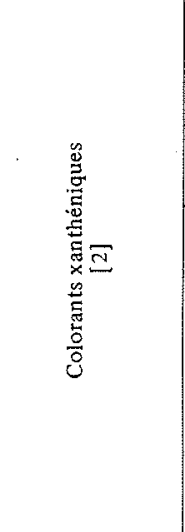 } & Anion & \multirow{5}{*}{\multicolumn{2}{|c|}{ 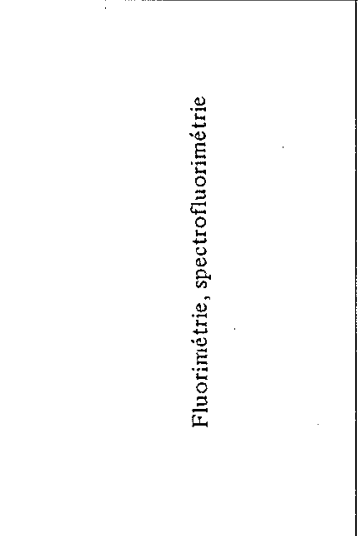 }} & \multirow{5}{*}{ 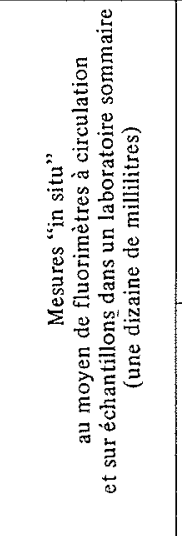 } & \multirow{3}{*}{$\simeq 10^{-11}$} & \multirow{5}{*}{ 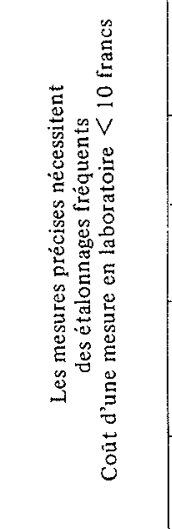 } & $\begin{array}{l}\text { Tous domaines à l'ex- } \\
\text { ception des milieux } \\
\text { fortement rétenseurs }\end{array}$ \\
\hline Rhodamine B & & Cation & & & & & & $\begin{array}{l}\text { Hydrologie de surface } \\
\text { (traceur médiocre mais } \\
\text { économique) }\end{array}$ \\
\hline Rhodamine $\mathrm{Wt}$ & & \multirow{3}{*}{ 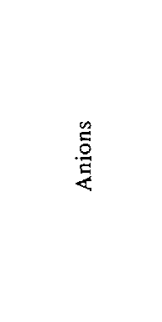 } & & & & & & $\begin{array}{l}\text { Tous domaines à l'ex- } \\
\text { ception des milieux } \\
\text { fortement rétenseurs }\end{array}$ \\
\hline $\begin{array}{l}\text { Sulforhodamine } \\
\text { B (rose sulfaci- } \\
\text { de brillant } \\
\text { Pontacy bril- } \\
\text { lant Pink B) }\end{array}$ & & & & & & $\simeq 210^{-11}$ & & $\begin{array}{l}\text { Hydrologie de surface } \\
\text { (traceur médiocre) }\end{array}$ \\
\hline $\begin{array}{l}\text { Sulforhodamine } \\
G\end{array}$ & & & & & & & & $\begin{array}{l}\text { Tous domaines à l'ex- } \\
\text { ception des milieux } \\
\text { foriement rétenseurs }\end{array}$ \\
\hline
\end{tabular}

[1] in Molinari (1969 b)

[2] in Molinari (1969 a) 
mises au point sur les traceurs, bien qu'à leur actif on puisse citer le dichromate mesuré par réactif coloré (André 1964) puis par absorption atomique (Burelli, Ecrément 1969), seul traceur utilisé systématiquement en hydrométrie (André 1969) et l'iode sous forme d'iodure, anion indiscutablement le plus performant en milieu poreux.

L'utilisation de ce dernier traceur, initiée par dosage radiométrique (Guizerix 1965) ou par radioactivation a pu être très largement développée par microdosage catalytique (Millet 1966, Godin/Archimbaud 1967, Molinari 1969 a) de même sensibilité $\left(10^{-9} \mathrm{~g} / \mathrm{g}\right)$ mais de coût infiniment plus faible.

A ces traceurs d'emploi extrêmement répandu, on peut associer le lithium utilisé maintenant en hydrométrie sous forme de chlorure. Ce cation mesuré par spectrométrie d'émission de flamme avec une sensibilité de $5.10^{-10} \mathrm{~g} / \mathrm{g}$ (Ecrément/Burelli 1975) doit sans doute à son faible encombrement stérique (Rochon 1976) sa faible rétention.

\section{Mise en ouvre des traceurs}

Naturalistes, chimistes, physiciens, se sont longtemps ignorés sans pour autant accorder toute l'attention nécessaire aux contraintes technologiques et économiques des hydrologues, aussi bon nombre d'investigations n'ont pas toujours abouti aux résultats escomptés.

La carence en anions salins utilisables comme traceurs (des halogénures, seul l'iodure présente des teneurs naturelles suffisamment basses pour être utilisé comme traceur) a conduit certains chercheurs à prôner fort judicieusement le recours à des cations chélatés sous forme de complexes anioniques, mais a proposer leur dosage par radioactivation dont le coût (sauf pour certains travaux exceptionnels) se révèle de toute évidence prohibitif, alors que ces mêmes complexes pourraient être aisément mesurés par spectrofluorimétrie.

En élaguant même quelques autres extravagances de la même veine (comme l'obstination de certains chercheurs à suggérer l'emploi de traceurs "neutrophages" qui requièrent des sources de neutrons pour une mesure imprécise et entachée d'erreurs) l'hydrologue dispose d'ores et déjà d'une gamme bien diversifiée de traceurs éprouvés.

Aux yeux du praticien, la facilité de détection peut revêtir une importance cruciale dans toutes les circonstances où les phénomènes à observer sont fugaces et constituent des éléments de décision (recherches itératives).

C'est en général le cas de l'hydrologie de surface pour l'étude des processus de dispersion où les traceurs mesurables par télé-détection (traceurs colorés et fluorescents) ou in situ (traceurs fluorescents et radioactifs) sont l'unique recours.

En fait, seuls les traceurs radioactifs émetteurs gamma, du fait de leur détection par sonde immergeable, autorisent de véritables mesures de concentration in situ ; cet avantage est exploité dans tous les domaines de l'hydrologie et notamment dans tous types de diagraphies.

Ces propriétes, ainsi que le pouvoir de pénétration du rayonnement, permettent, grâce à des générateurs de radioéléments, la mesure directe des débits de canalisations en charge (conduites forcées) et ont fait entrevoir la possibilité de mesures automatiques de débit en écoulement libre, mesures qui peuvent également être envisagées au moyen de traceurs fluorescents.

Hormis ces deux familles de traceurs, il n'est pas d'exemple courant de mesure in situ d'autres traceurs pour lesquels il est toujours nécessaire de procéder à des prises d'échantillons, qui, pour la plupart, sont de volume extrêmement réduit et n'obèrent en rien le déroulement des essais.

\section{Besoins nouveaux et perspectives de développement des traceurs}

Deux voies nouvelles s'offrent aux recherches futures, l'une est dictée par le double souci d'augmenter l'efficacité des essais et de réduire le coût des interventions.

L'impossibilité de maîtriser la durée d'essais de traceurs, durée imposée par la nature des processus étudiés, incite à tirer parti de l'intervention pour procéder à des essais multiples. Ces multitraçages qui présentent un intérêt évident dans tous les domaines (hydrologie de surface, karst, hydrologie souterraine) requièrent la mise au point de nouveaux traceurs compatibles entre eux et dotés de qualités analogues aux traceurs déjà éprouvés avec succès dans tous ces milieux.

La seconde voie d'investigations concerne les études de propagation de substances polluantes, études qui font appel à double titre aux techniques de traceurs puisqu'elles impliquent à la fois une bonne connaissance des processus de transfert $d u$ fluide vecteur et des processus d'interaction des substances incriminées (dont les concentrations en général faibles se situent d'ailleurs à un niveau similaire à celui des traceurs employés). En fait, puisque du point de vue des interactions, rien ne distingue un traceur d'une substance polluante (et nos propres travaux ont effectivement montré que, dans certains milieux, des substances polluantes pouvaient être moins sorbées que des substances réputées bon traceurs), apparait de toute évidence la nécessité d'appréhender d'emblée l'ensemble des mécanismes de transfert dans la perspective d'une modellisation des processus de convection (transfert $\mathrm{du}$ fluide vecteur) et des processus d'interaction établie sur un grand nombre d'essais faisant intervenir diverses substances-type.

\section{Références}

ANDRÉ (H.) - Hydrométrie pratique des cours d'eau - Jaugeages par la méthode de dilution. Electricité de France - Division Technique Générale du Service de la Production Hydraulique Grenoble 1964 - 88 pages

ANDRÉ (H.) - Méthodes par traceurs utilisés en 1969 pour la mesure des débits des liquides - La Houlle Blanche, $1969, \mathrm{n}^{\circ} 5$, pp. 535-539

BATSCHE (H.), BAUER (F.) BEHRENS (H.), BUCHTELA (K.), HRIBAR (F.), KÄSS (W.), KNUTSSON (G.), MAIRHOFER (J.), MAURIN (V.), MOSER (H.), NeUmaIER (F.), OSTANEK (L.) RAJNer (V.), RAUERT (W.), SAgl (H.), Schnitzer (W.A.), ZÖTL (J.) - Essais comparés de traçages dans le karst en moyenne Styrie en 1966. Steïer Beitrag. Hydrogeol. n ${ }^{\circ} 19, \mathrm{pp}, 331-403$ Traduction $n^{\circ} 5271$ du BRGM.

BURELLI (F.P.), ECREMENT (F.) - La détermination du chrome dans les eaux par spectrométrie d'absorption atomique - Application aux mesures de débit de cours d'eau.Chim. Analytique $1969,51,561$

DOMBRowski Von. (H.) - Die Anwendbarkeit der hydrogeologischen Markierungsmittel zur Untersuchung der Beziehungen Zwischen Grundwasser und oberirdischem Wasser -. Markierung mit Serratia marcescens - Z. deutsch geol. Ges. Sonderh. Hydrogeol. Hydrogeochem. S 117-118 Hannover 1970

ECREMENT (F.), BURELLI (F.P.) - Détermination du Lithium dans les eaux par spectrométrie d'absorption atomique et d'émission de flamme - Application : utilisation du lithium comme traceur en hydrologie -Analusis, 1975, V 3, n³, pp. 146 à 156 
FrantisaK (F.), PALAdE dE IRIBARNE (A.), SMith (J.W.) HUMMEL (R.L.), Nondisturbing tracer technique for quantitative measurements in turbulent flow - Industrial and Engineering chemistry Fundamental, 1969 , Vol. 8, n 1, pp. 160-167

GODIN (J.M.), ARCHIMBAUD - Microdétermination rapide de l'lode dans les Eaux-Commissariat à l'Energie Atomique Centre de Pierrelatte, Rapport CEA-R-3145, 1967

GUIZERIX (J.) - Travaux non publiés - Commissariat à l'Energie Atomique Centre d'Etudes Nucléaires de Grenoble - 1965

HOROWITZ (A.) - Palynological tracing of saline water sources in lake Kinneret region (Israel), $J r$. of Hydrology, 10, 1970,pp. 177-184

MLlet (M.) - Travaux non publiés. Commissariat à l'Energie Atomique. Centre d'Etudes Nucléaires de Grenoble. 1966.

MOLINARI (J.) - Les traceurs salins ef fluorescents en hydrologie. Aspects techniques et économiques de leur utilisation - Commissariat à l'Energie Atomique - Centre d'Etudes Nucléaires de Grenoble, Rapport DR/SAR-G/69-15, Juin 1969, 36 pages.
MOLINARI (J.) - L Les traceurs isotopiques en hydrologie - Annales. des Mines, Octobre 1969, pp. 13-38

PALOC (H.) -- Les Traceurs en hydrogéologie karstique. Leur apport à la connaissance des réservoirs aquifères calcaires -.- Institut National des Sciences et Techniques Nucléaires - Session d'Information sur l'emploi des traceurs en hydrologie, en sédimentologie et en génie civil. Saclay Avril 1975, BRGM -... Centre Scientifique d'Orléans La Source 45018 Orléans Cedex.

ROCHON (J.) -- Commissariat à l'Energie Atomique - Centre d'Etudes Nucléaires de Grenoble -... Thèse 1976 (à paraître)

ROUCH (R.) -- Le système karstique du Baget. Etude des Harpacticides rejetés au niveau de Las Hountas au cours de plusieurs crues du cycle hydrologique 1970-1971 -. Annales Spéléol. 1972 27,1, pp. 139-176

SCHNITZER (W.A.), WAGNER (W.) - Welche Anforderungen müssen Geruchsstoffe für Karstwassermarkierungen erfüllen ? Wasser $1969-8$, pp. $227-236$

\section{Discussion}

Président : M. Pierre LEVEQUE

M. Le Président remercie M. Molin A R I pour son exposé qui donne un excellent panorama des travaux récents concernant le mécanisme des interactions susceptibles d'affecter le comportement des traceurs. Il ouvre ensuite la discussion.

M. FONTES souligne l'importance de la phase argileuse au contact ou en suspension dans l'eau, dans toutes les expériences comportant l'emploi de traceurs. D'une argile à l'autre les échanges d'ions (adsorption et piégeage) sont très différents. D'où l'intérét d'une étude conjuguée du traceur et du milieu -en particulier lorsque l'on emploie le lithium-dans les expériences exigeant une certaine finesse.

C'est là, répond M. MOLINARI, une bonne introduction à l'exposé que je dois faire cet après-midi sur les processus de transport en milieu poreux. Dans ce domaine, nous avons rencontré les problèmes signalés par M. FONTES et tenté de les résoudre -en praticiens-par la méthode de la "boîte noire" qui nous est assez familière.

M. Pierre LEVEQUE donne un très original exemple de la rétention de certains ions par les argiles : celui du "réacteur naturel et fossile" découvert en 1972 dans le gisement d'uranium d'Oklo $\left({ }^{1}\right)$ au Gabon. Ce réacteur naturel a fonctionné pendant 800000 années environ, il y a de cela un bon milliard d'années.

Les éléments de fission qui se sont formés au cours de cette longue période ont été absorbés par le sol et ne se sont pas déplacés d'un micron cela témoigne d'un potentiel de rétention extraordinaire. Le plutonium, en particulier, n'a pas changé de place mais s'est transformé en uranium 235. A Oklo, la nature semble nous offrir une réponse rassurante à la question que se pose parfois le public à propos du stockage souterrain des déchets radioactifs : peut-on garantir que dans plusieurs siècles les produits radioactifs n'auront pas "migré"?

Effectivement commente $M$. Le Président, les forces de VAN DER VALS constituent un domaine de "recherche fondamentale" très intéres sant dont les applications ne se limitent pas à l'adsorption des ions par les argiles. Ainsi, on a constaté que le graphite -dont les "plans" ne sont liés que par les dites forces- pouvait constituer un véritable réservoir a cessium.

Madame A. BERTHOME, qui habite sur les bords de la Loire, désirerait savoir si la flore et la faune de ce fleuve risquent de souffrir du réchauffement de l'eau dû aux centrales nucléaires implantées sur ses rives.

Ce problème d'ordre biologique ne relève pas du genre d'investigation que nous poursuivons, répond M. MOLIN A RI, mais les techniques de traceur permettent de suivre le cheminement et l'évolution de diverses

(1) Voir "Le phénomène d'Oklo" par R. NAUDET, Revue générale nucléaire, février-mars 1975 , pages $45-50$ (N.D.L.R.). substances véhiculées par l'eau. M. GUIZERIX, M. MARGRITA et moimême, en donnerons des exemples dans la communication que nous présenterons.

M. Le Président clót la discussion et donne la parole successivement à M. FONTES et à M. le Professeur LETOLle pour l'exposé de leur communication.

M. le Président BANaL profite de l'intervalle entre l'exposé des deux demières communications de la matinée pour présenter M. CAZENAVE, son successeur à la Présidence du Comité technique, empêché d'assister au début de la séance par des obligations professionnelles inopinées.

Sur l'invitation de M. BANAL, M. CAZENAVE prononce ensuite l'allocution suivante :

"Je suis extrêmement honoré de la confiance que vous me témoignez en me choisissant comme President de ce Comité. Je suis aussi très impressionné : d'abord, parce que je vais me trouver à la tête de votre groupe qui, comme le disent fort justement les statuts de la S.H.F. "rassemble les spécialistes les plus qualifiés de l'Université, des Administrations et de l'Industrie" ; mais aussi, parce que je fais suite dan cette fonction à des personnalités tout à fait éminentes, auxquelles je n'avais jamais envisagé, ni même imaginé, de succéder. Pour ne pas concentrer sur le seul Président BANAL, qui m'en voudrait un peu, le feu de mes éloges, je rappellerai que la fonction qui m'est aujourd'hu offerte a été assurée, et avec quel brio, pendant presque vingt ans par le Président BARRILLON, qui institua un rythme de Sessions qui est encore en usage ; il donna d'emblée à la S.H.F. la grande diversification d'activités qui constitue son image de marque la plus originale et sa qualité la plus fondamentale. Avec l'universalité de ses connaissances et de sa pensée, et la grande faculté de synthèse entre des sciences apparemmen dispersées qui est l'apanage des très grands esprits scientifiques, M. Robert GIBRA T ne pouvait qu'étendre encore le champ de vision de notre Société. Enfin, le Président BANAL, nourri d'une expérience de très haut niveau dans tous les domaines de l'hydraulique, assure depuis dix ans avec l'attention précise et l'autorité toujours souriante qui sont l'expression de sa très sûre et très profonde compétence, des activités toujours aussi régulières malgré leur diversification de plus en plus étendue, et coordonne l'action d'un nombre considérable de Divisions et de Commissions : remarquablement organisées, celles-ci constituent un appareil d'étude que beaucoup de sociétés scientifiques aimeraient avoir Après de si brillantes réussites et devant une telle ampleur et une telle qualité d'activités, je suis naturellement pris de vertige, d'autant que je prends la barre à un moment où, du fait de la disparition presque totale 
des sites français, l'hydroélectricité qui fût, au départ, l'axe principal d'activité de la S.H.F., doit continuer de laisser à d'autres techniques la place qui fut longtemps la sienne, sans que pour autant la S.H.F. cesse d'être la très efficace tribune d'échange entre les ingénieurs de cette technique, technique qui, en s'expatriant, se lance dans des mutations et des extrapolations qui montrent sa vitalité et dans lesquelles l'ingéniérie française garde une place de premier plan. Il nous faudra donc trouver en ce domaine un mode d'expression nouveau, tenant compte à la fois de la décroissance de son poids économique en France et de l'ingéniosité persistante de ses techniques. Mais, plus que jamais, le champ d'action de la S.H.F. doit continuer de s'étendre, et nous devons avoir le souci d'être un carrefour de réflexion entre chercheurs et ingénieurs dans toutes les techniques où le fluide joue un rôle de puissance ou de contrôle, dans toutes celles où la présence, ou l'absence, ou le changement d'état d'un ou plusieurs fluides peut être bénéfique ou utile, et encore dans tous les domaines où l'importante méthodologic mathématique, physique ou hybride mise au point par les hydrauliciens peut aider au progrès d'autres sciences ou d'autres techniques.

“Telle est la voie tracée par mes prédécesseurs et que je m'efforcerai de suivre. C'est un objectif qui ne peut s'atteindre qu'en équipe, et je compte, à la fois, sur l'appui de mes aînés et du Conseil d'Administration, sur l'active coopération du Bureau et des Commissions et sur l'extraordinaire force vive que constitue le Comité technique. C'est donc vers vous Messieurs que je me toume en faisant pour terminer un appel très pressant à votre aide précieuse et dynamique". 